\title{
Observatorio
}

\section{Bibliotecas universitarias: ¿sabemos medir sus resultados e impactos?}

\author{
Por Atilio Bustos-González
}

Resumen: Las bibliotecas deben tener sistemas para medir su rendimiento y poder demostrar así que son útiles para sus instituciones. Se analizan los aspectos a considerar: objetos de aprendizaje, repositorios de información científica, investigación, producción científica, biblioteca digital, etc. Se describen brevemente los resultados de la Red ALFA - Biblioteca de Babel: 1. Anuario de bibliotecas universitarias, 2. Directrices para la creación de los repositorios institucionales, y 3. Estrategias didácticas para el uso de las TICs en la docencia. Aunque hallar buenos indicadores no es trivial, el autor ofrece una tabla de ellos.

Palabras clave: Indicadores de cobertura, Indicadores de eficiencia, Indicadores de eficacia, Objetos pedagógicos, Repositorios, Producción científica, Estadísticas, Política universitaria

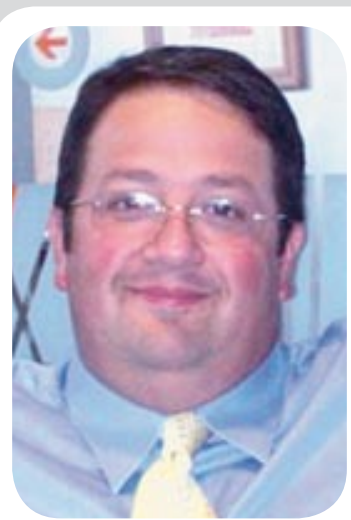

Atilio Bustos González es licenciado en Bibliotecología, por la Univ. de Chile en 1983 con máxima distinción. Cursó estudios de postgrado en Administración de Empresas entre 1984 y 1986, y de Maestría en Gestión y Política Universitaria en la Universidad Politécnica de Cataluña en Barcelona entre 2002 y 2004. Desde 1995 es Director del Sistema de Biblioteca de la Pontificia Universidad Católica de Valparaíso. Ha trabajado como consultor de organismos internacionales $y$ nacionales, desarrollando misiones en Argentina, Bolivia, Brasil, Chile, Colombia, Ecuador, México, Nicaragua, Perú, Uruguay y Venezuela. Ha dictado cursos en más de 40 universidades latinoamericanas. Posee una amplia experiencia práctica y docente en los diversos aspectos de la gestión de bibliotecas universitarias, en edición de revistas científicas y publicaciones académicas.

\section{Title: University libraries: Can we measure their outcomes and impact?}

Abstract: University libraries need ways of measuring their effectiveness as a means of demonstrating their value to their institutions. The roles that could be considered include: learning objects, repositories of scientific information and research, digital library, etc. These aspects are analyzed and the findings of the ALFA Network's Biblioteca de Babel project are briefly described. The Babel report has three elements: 1. Annual report on university libraries, 2. Guidelines for creating institutional repositories, and 3. Teaching strategies for the use of Technologies of Information and Communication (TICs) in education. Although it is not easy to find good indicators, the author offers recommendations.

Keywords: University libraries, Coverage indicators, Efficiency indicators, Effectiveness indicators, Pedagogical tools, Learning Objects, Repositories, Scientific output, Statistics, University policy

Bustos-González, Atilio. "Bibliotecas universitarias: ¿sabemos medir sus resultados e impactos?”. En: El profesional de la información, 2007, julio-agosto, v. 16, n. 4, pp. 281-286.

DOI: 10.3145/epi.2007.jul.01

ESTOY PREOCUPADO. No tenemos un termómetro adecuado para medir el impacto de las bibliotecas universitarias. Esta ausencia es evidente para quien dé una ojeada a los anuarios estadísticos de asociaciones de bibliotecas universitarias de América y Europa. Muestran una disminución de los préstamos físicos, una merma en la tasa de rotación de la colección monográfica tradicional, una disminución de la tasa de préstamos por funcionario, $y$ un aumento en el costo por consulta. Muchos de los libros mueren de obsolescencia en las estanterías, antes de ser prestados la suficiente cantidad de veces como para amortizar su adquisición. Los libros digitales apenas son consultados.
En este observatorio no se discute el grado de adaptación de las bibliotecas universitarias a la transición de un mundo impreso a uno digital. Todas parecen estar haciendo sinceros esfuerzos por realizar este cambio. La preocupación que expreso es más permanente. No estaba resuelta en la biblioteca de la Galaxia Gutenberg, y hasta donde hemos podido ver, tampoco está solucionada para la biblioteca universitaria de la Sociedad del Conocimiento.

La preocupación que deseo destacar es que si no contamos con un adecuado instrumental para medir los resultados e impactos de las bibliotecas en las universidades, difícilmente podemos estar seguros de si apor- 
tamos valor a la institución de la que formamos parte. Si un organismo no aporta valor al cuerpo, se vuelve prescindible, y si tiene suerte sólo se hipertrofia. ¿Queremos este futuro para las bibliotecas universitarias?, ¿estamos haciendo lo suficiente para subsanar este déficit?, ¿la solución vendrá de otra parte?

\section{Los indicadores de gestión, ¿miden lo que deben medir?}

Estimado lector: le invito a que se aleje por unos minutos de su muy respetable puesto en la biblioteca o centro de documentación y se localice en el despacho de un vicerrector o de un director. Seguramente desde esta posición de gestión-política y atendiendo a la cuantía de los recursos implicados, le surgirán dudas respecto del beneficio de la inversión realizada en información y en general en las bibliotecas. Probablemente se hará preguntas relacionadas con el coste-oportunidad y otras como: ¿dónde es más beneficioso invertir un euro adicional?, ¿están dando frutos suficientes los dineros invertidos?, ¿apoyan estos recursos la innovación y la construcción de un Espacio de Educación Superior? Las bibliotecas no son capaces de medir cuáles son los impactos que los medios puestos a su disposición generan sobre la capacidad de investigar o de mejorar la calidad de los aprendizajes de su comunidad. No contamos con un instrumental adecuado que permita dar certeza a nuestros directores que la inversión realizada rinde objetivamente frutos ciertos, valiosos y cuantificables.

\section{"Por definición, los resultados siempre se producen dentro de la organización y los impactos fuera de ella. La frontera en este caso es la puerta de la Biblioteca. Por lo tanto los impactos son el grado de contribución de las bibliotecas universitarias al logro de la misión y los objetivos de la Universidad en su conjunto"}

$\mathrm{Si}$ analizamos el panorama de los anuarios estadísticos antes señalados desde un punto de vista de la teoría de sistemas, descubriremos que existe un gran interés por medir insumos (inputs), un interés relativamente bajo por medir procesos, y un interés moderado por medir resultados. Descubrimos que el instrumental de indicadores es abundante en medidas de eficacia, y moderado en proveer medidas de eficiencia y cobertura. No ha sido posible encontrar indicadores de impacto.

\section{Objetos de aprendizaje y repositorios de información científica}

Una segunda mirada a los anuarios estadísticos permite hacer una lectura de los temas ausentes. Es fácil darse cuenta que éstos no ofrecen medidas sobre los objetos de aprendizaje, tan frecuentemente usados por los profesores y alumnos. Tampoco ofrecen medidas respecto de los repositorios de información científica. En verdad los anuarios estadísticos casi no son capaces de proveer indicadores sobre las bibliotecas digitales. Nuevamente, descubrimos que no tenemos termómetro.

Estas dos grandes tipologías de objetos documentales, gestionados de un modo particular (metadatosgestión distribuida en distintas topologías), cobran un sentido especial en las bibliotecas universitarias de hoy. Los objetos pedagógicos ofrecen la oportunidad de suministrar contenidos altamente pertinentes para el aprendizaje de los alumnos. Influyen en la calidad, velocidad y pertinencia de los aprendizajes de los estudiantes. Por otra parte, la producción científica, pone a la biblioteca universitaria en condiciones de contribuir activamente a dar visibilidad al conocimiento generado en la propia institución.

La administración de repositorios es una tarea del presente y futuro. No da lo mismo que lo asuma un campus virtual, la unidad de informática o la biblioteca. Son roles diferentes. La misión de la biblioteca es, entre otras, administrar y proveer, independientemente de su origen autoral, acceso a los recursos de información de calidad que la comunidad requiere. En este contexto es fundamental garantizar que cualquier miembro de la comunidad pueda acceder sin trabas a los recursos de información que le parezcan pertinentes para atender su necesidad de saber. En la lógica de las aulas virtuales, éstas se abren junto con el inicio de un curso y se cierran al terminar el mismo. Sólo pueden ingresar y acceder a esos recursos los alumnos matriculados en el curso. Esta diferencia entre el libre acceso y el acceso restringido, en nuestra opinión fuerza a la biblioteca universitaria a hacerse cargo la gestión de los objetos de aprendizaje, los que en su conjunto conforman la biblioteca virtual de pregrado.

Hoy, como nunca antes en la historia de las bibliotecas universitarias, éstas pueden asumir un rol activo sobre dos funciones fundamentales del quehacer de sus instituciones: gestión del aprendizaje y gestión del conocimiento. Al hacer esto, estarán aportando valor a la institución que las cobija, en un conjunto de tareas que otros organismos internos no pueden hacer con igual nivel de eficiencia. De este modo las bibliotecas pueden provocar impactos positivos. 


\section{Buscando respuestas en conjunto}

He tenido el honor de formar parte de la Red ALFA. Biblioteca de Babel, una iniciativa apoyada por la Oficina de Cooperación de la Unión Europea. La Red creada en París en 2005, está integrada por 9 universidades de Europa, 15 de Latino América y la Asociación Columbus. Su propósito ha sido estudiar, sistematizar y documentar cómo las universidades de Europa y América Latina están integrando en sus servicios de biblioteca las nuevas prácticas pedagógicas basadas en las tecnologías de la información y comunicaciones (TICs), identificando metodologías para desarrollar esta integración y estrategias para la evaluación de los impactos alcanzados sobre la calidad de la docencia universitaria y el aprendizaje.

Mediante un mecanismo de intercambio de buenas prácticas, se generó un flujo muy positivo de diálogo norte-sur, donde ambos hemisferios pudieron aportar y recibir. Hoy están en prensa dos de sus productos, y en pocas semanas estarán disponibles en el sitio web del proyecto, que obviamente es un repositorio de objetos de aprendizaje llamado Biblioteca de Babel (ver Pantalla 1).

Biblioteca de Babel generó tres productos:
- Anuario estadístico y de indicadores de las bibliotecas universitarias participantes en la Red ${ }^{1}$,

- Directrices para la creación de los repositorios institucionales en universidades y organizaciones de educación superior, $\mathrm{y}$

- el manual Estrategias didácticas para el uso de las TICs en la docencia universitaria presencial.

El Anuario de bibliotecas universitarias propone nuevos indicadores que permiten superar algunas de las deficiencias antes expresadas:

http://agora.ucv.cl/ens.php? $e=105$

En la tabla 1 se despliegan indicadores de insumo, proceso, resultado e impacto. A su vez se realiza una segunda taxonomía por indicadores de cobertura, eficiencia y eficacia. Se destaca con color de fondo sobre la cruz aquellos indicadores clave que permiten generar un tablero de control y reflejan de una forma sintética el estado de una biblioteca universitaria.

En los indicadores de impacto se quiso introducir una mirada desde el ISI-Web of Science y desde Scopus. Esta segunda, es una base de datos con una cobertura más equilibrada que refleja de mejor modo la producción científica de calidad de todos los continentes. Sin

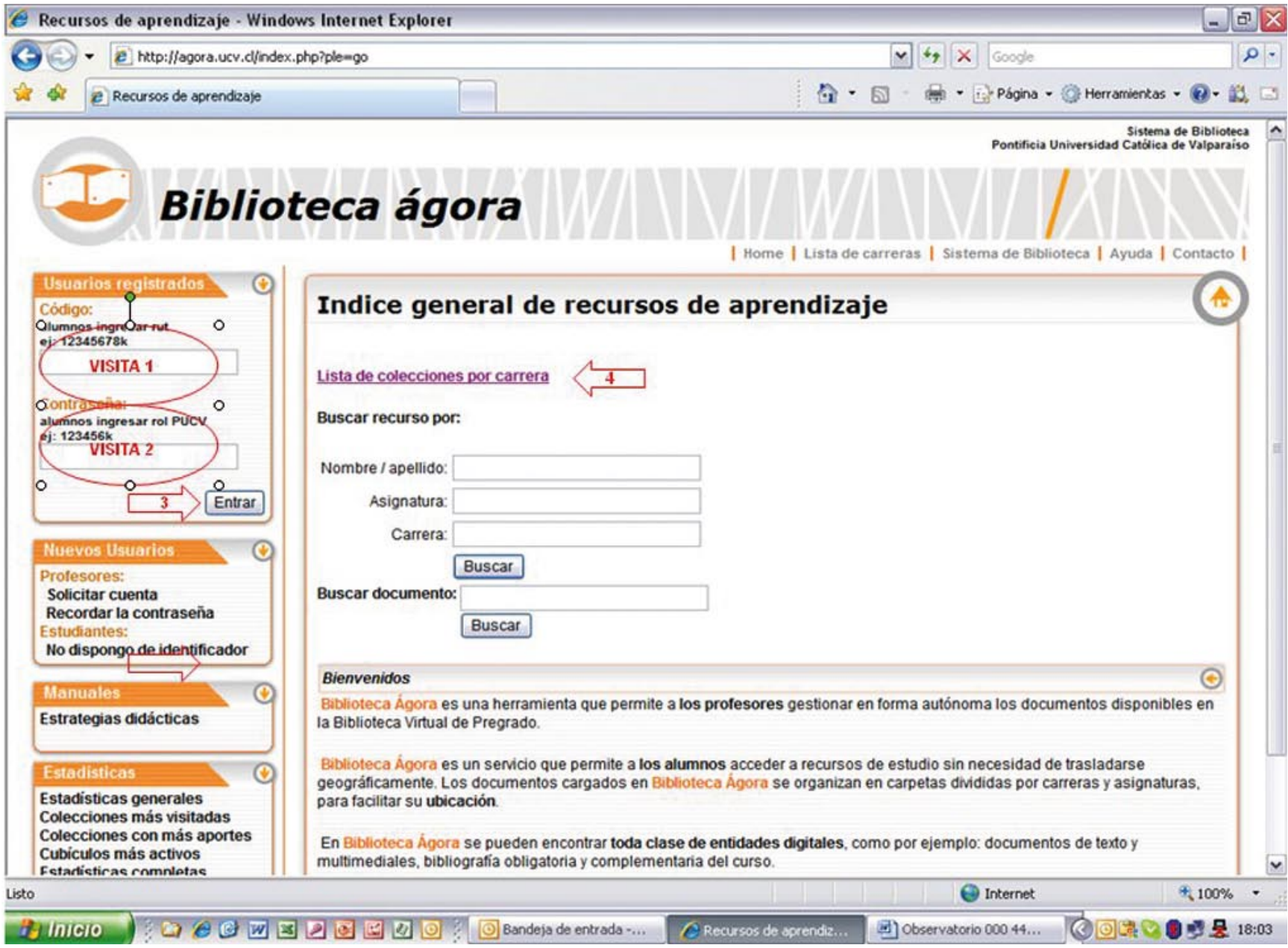

Pantalla 1. Repositorio Biblioteca Ágora 


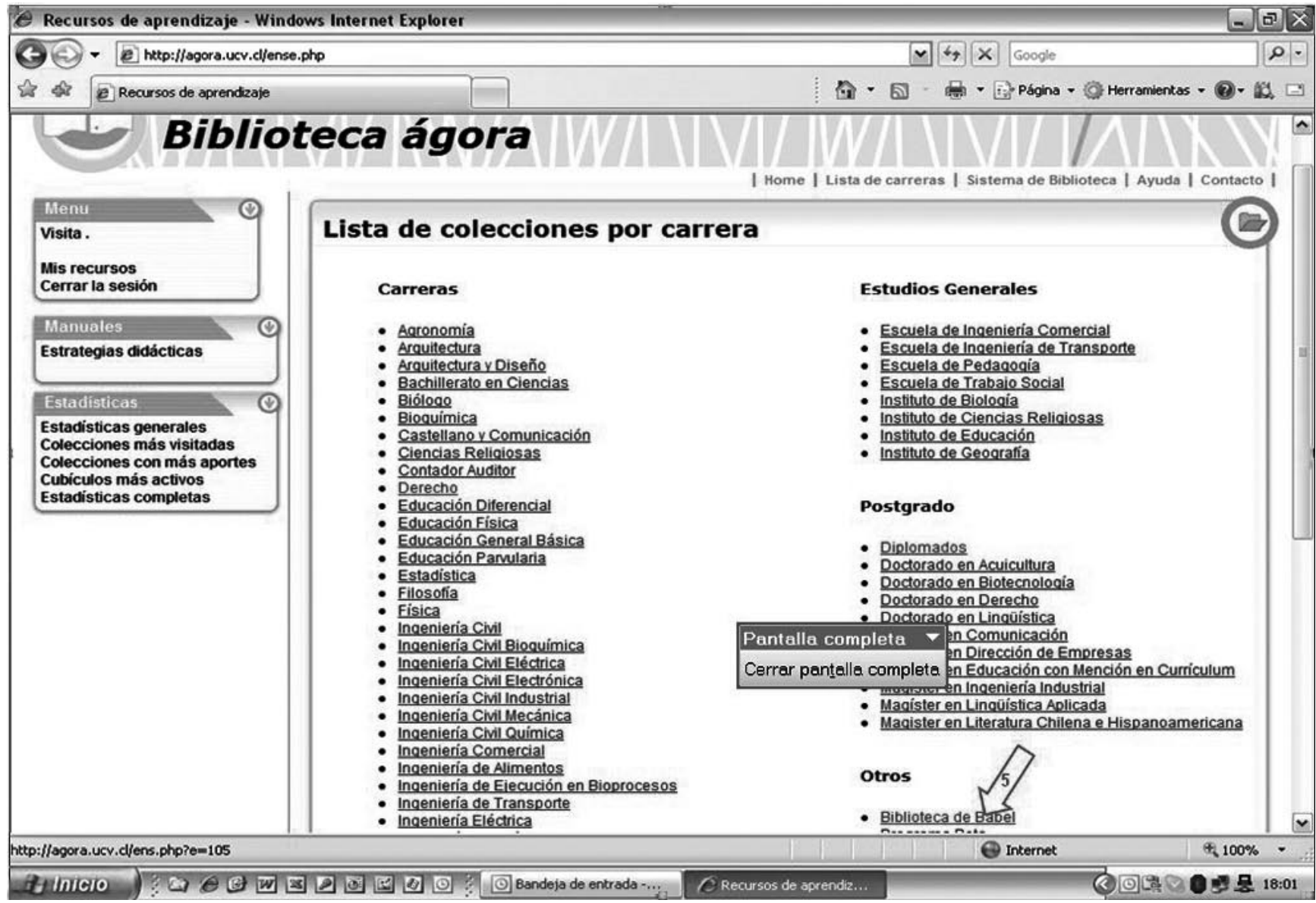

Pantalla 2. Repositorio Biblioteca de Babel

embargo, respecto a indicadores de impacto el camino por recorrer aún es largo, y sólo se presenta una propuesta inicial.

El segundo producto citado, Directrices para la creación de los repositorios institucionales en universidades y organizaciones de educación superior, puede consultarse en:

http://agora.ucv.cl/docs/651/Manual_despues_valparaiso_revision_4.doc

El repositorio institucional se entiende como un sistema de información que reúne, preserva, divulga y da acceso a la producción intelectual y académica de las comunidades universitarias. En la actualidad el repositorio institucional se constituye en una herramienta clave de la política científica y académica de las universidades. Por otro lado, el acceso al texto completo de los objetos de aprendizaje hace que el repositorio se constituya en una pieza fundamental de apoyo a la enseñanza y a la investigación, a la vez que multiplica la visibilidad institucional de la comunidad internacional. Dentro de este escenario las bibliotecas universitarias son el órgano que, por su experiencia en la gestión de la información en todas sus formas y el contacto con el conocimiento, deberán liderar la implementación de

\section{Repositorios universitarios}

Entre algunos pioneros del acceso abierto, los que crearon repositorios temáticos como ArXiv (física, matemáticas, informática...), RepEc (economía) , E-LIS (biblioteconomía, documentación y archivística)..., hubo cierta desazón con el modelo actual de que cada universidad esté poniendo en marcha su propio repositorio. Ello significa consumar la dispersión de documentos multidisciplinares, algo que por otro lado tampoco sería grave si los documentos estuvieran bien indizados y pudieran ser localizados por los harvesters (recolectores) o buscadores.

Independientemente de la fácil localización o no de los documentos, el aspecto claramente positivo de los repositorios institucionales es que ayudan (u obligan, según la política de la institución) a mentalizar a los autores y -gracias casi siempre a la acción de los bibliotecarios del lugar-afloran en la Red documentos que de no ser por el repositorio institucional quizá no se hubieran visto nunca.

Por último, los documentos también pueden depositarse, además, en los repositorios temáticos. 


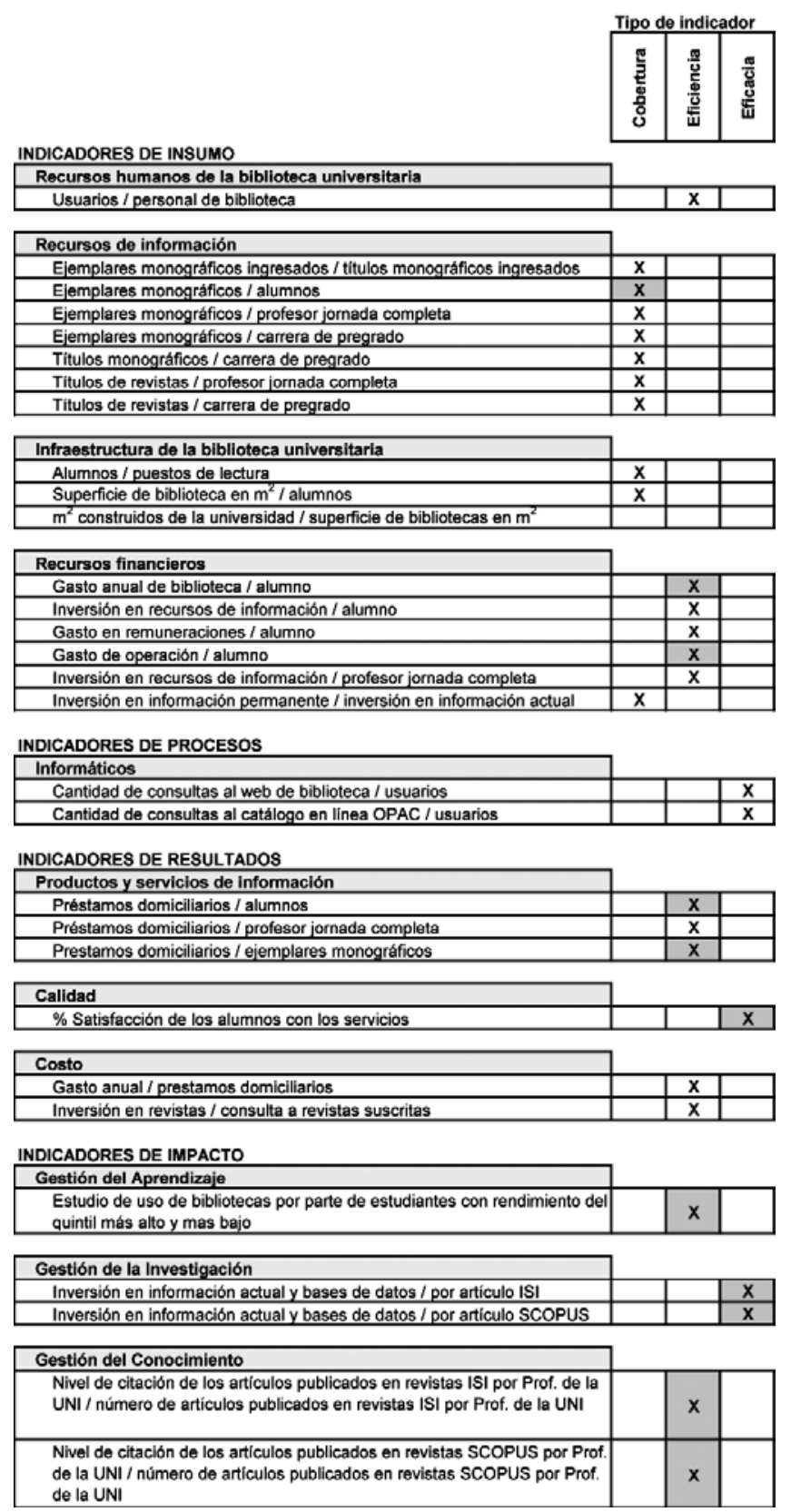

Tabla 1: Indicadores para bibliotecas universitarias Fuente: Red ALFA Biblioteca de Babel

los repositorios institucionales con el fin de lograr la competitividad educativa.

El tercer producto es el manual Estrategias didácticas para el uso de las TICs en la docencia universitaria presencial

\section{http://agora.ucv.cl/babel/}

Su objetivo es poner a disposición de profesores, estudiantes y bibliotecarios un conjunto de estrategias metodológicas que faciliten la integración de las nuevas tecnologías de información y comunicación (TICs) en los procesos docentes presenciales. La utilización de este manual no requiere de una plataforma de aprendizaje específica, posibilitando realizar actividades desde el repositorio de objetos de aprendizaje, hasta la utilización de plataformas de e-learning.
El manual se divide en dos apartados:

1. Se presentan algunos elementos de la acción docente universitaria en la Sociedad del Conocimiento. Analiza los cambios sociales, laborales y educativos que las TICs están generando. Revisa en forma sintética cómo las teorías del aprendizaje se hacen cargo de estas transformaciones y cómo, ellas a su vez generan dentro de las universidades un profundo cambio del papel del estudiante, del profesor, de los recursos didácticos y de las bibliotecas. A esta sección se accede por un mapa conceptual.

2. Corresponde a lo que se denominó "recetas metodológicas", que son un conjunto de componentes didácticos que abarcan el empleo docente de herramientas tecnológicas, metodologías de enseñanza activa, actividades docentes e indicaciones para la generación de materiales de apoyo.

2.1. Estas recetas metodológicas son la primera de las formas de acceder a los contenidos de este apartado.

2.2. La segunda forma corresponde a lo que se ha denominado línea del tiempo, ofreciendo el acceso a diferentes componentes didácticos de acuerdo con el grado de avance del período lectivo.

2.3. La tercera forma de acceder a los contenidos es por grado de dificultad de los diferentes componentes didácticos que se ofrecen.

2.4. Obviamente, la cuarta alternativa corresponde a la lectura secuencial de ellos.

Todas estas opciones buscan ofrecer una forma sencilla, pertinente y oportuna de aproximarse a los diferentes componentes propuestos. Cada componente didáctico mantiene una estructura común, incluida una interrelación de la receta metodológica reseñada con otras que se recomienda desarrollar en forma previa, paralela $\mathrm{o}$ a posteriori.

\section{Una reflexión abierta}

La tarea de generar indicadores de gestión de las bibliotecas universitarias que den cuenta de los impactos no es trivial. Se trata de aislar unas variables complejas, que se entrelazan con las acciones de otros actores interesados en mejorar la calidad de los aprendizajes y de generar la más alta visibilidad para la producción científica institucional. Esta es una invitación a un diálogo, a una reflexión en conjunto y a un intercambio de buenas prácticas. Son bienvenidas las críticas, así como las propuestas.

Seguramente deberemos ser capaces de construir indicadores complejos, de segunda generación, usando nuevas formas de representación gráfica, donde sea po- 
sible cruzar cuatro o más variables y en forma animada mostrar tendencias intertemporales. La cienciometría, la infometría, el datamining podrán aportar instrumental para avanzar en esta tarea.

Lo que nos queda claro es que si nosotros no somos capaces de medir el impacto de nuestra tarea y el grado de contribución de las bibliotecas universitarias a los objetivos institucionales, estamos en una posición muy débil de cara al futuro. El mundo es lo suficientemente competitivo y la gestión universitaria se ha profesionalizado lo suficiente como para que las viejas declaraciones poéticas sigan teniendo efecto sobre los asignadores de recursos.

\section{Nota}

1. El diseño de nuevos indicadores tiene origen el año 2005 con el trabajo de una subcomisión del la Comisión de Directores de Bibliotecas de Universidades del Consejo de Rectores de Chile, el cual sirvió de antecedente para la elaboración de la propuesta de la Tabla 1.

\section{Bibliografía}

Ahumada, L.; Bustos-González, A. "Management of knowledge, information and organizational learning in university libraries". En: Libri, 2006, v. 56, pp. 180-190.

Association of College \& Research Libraries. Task force on academic library outcomes assessment report. Consultado en: mayo de 2007.

http://www.ala.org/ala/acrl/acrlpubs/whitepapers/taskforceacademic.htm

Association of College \& Research Libraries. Academic library statistics. Consultado en: mayo de 2007.

http://www.ala.org/ala/acrl/acrlpubs/acadlibrarystats/academiclibrary.htm

Association of Research Libraries. Consultado en: mayo de 2007.

http://www.arl.org/stats/initiatives/

Biblioteca Ágora. Consultado en: mayo de 2007.

http://agora.ucv.cl

Bibliotheksindex. BIX-WB: library index for academic libraries. Consultado en: mayo de 2007.

http://www.bix-bibliotheksindex.de/

Brophy, P. Measuring library performance: pinciples and techniques. London: Facet, 2006. 242 pp.

California State Library. Outcome measurement information. Consultado en: mayo de 2007.

http://www.library.ca.gov/html/grants.cfm

CD-LOR Project. Consultado en: mayo de 2007.

http://academy.gcal.ac.uk/cd-lor/index.html

Davis, H.; Somerville, M. "Learning our way to change: improved institutional alignment". En: New Library World, 2006, v. 107, n. 3/4, pp. 127-140. DOI: 10.1108/03074800610651907

DigiQUAL. Consultado en: mayo de 2007.

http://www.digiqual.org/

Fondren Library of Rice University. Consultado en: mayo de 2007. http:// www.rice.edu/fondren/custsrvc/vssurveyl.pdf

Grupo Scimago. "Ranking de instituciones de investigación iberoamericanas (RI3)". En: El profesional de la información, 2007, mayo-junio, v. 16, n. 3, pp. 258-260. DOI: 10.3145/epi.2007.may.10

Kezar, A. "Consequences of radical changes in governance: a grounded theory approach". En: The journal of higher education, 2005, v. 76, n. 6 , pp. 634-668.
LibQUAL+. Consultado en: mayo de 2007.

http://www.libqual.org/

Library Research Service. Consultado en: mayo de 2007. http://www.lrs. org/academic.php

Ludwig, L.; Starr, S. "Library as place: result of a delphi study". En: $J$. Med. Libr. Assoc., 2005, v. 93, n. 3, pp. 315-326.

Margaryan, A.; Milligan, C.; Douglas, P. Community dimensions of learning object repositories. Deliverable 9. Structured guidelines for setting up learning object repositories. Draft version. UK: CD-LOR Project, 2007. 17 p. Consultado en: mayo de 2007.

http://academy.gcal.ac.uk/cd-lor/documents/CD-LOR_Structured_Guidelines_v1p0_001.pdf

Medical Libary Association. Consultado en: mayo de 2007.

http://www.mlanet.org/resources/bench05/

IFLA. Statistics and evaluation section. Consultado en: mayo de 2007. http://www.ifla.org/VII/s22/statlinks.htm

National Library Statistics. Consultado en: mayo de 2007.

http://www.lrs.org/aca_nat.php

Pontificia Universidad Javeriana de Colombia. Biblioteca de Babel. Consultado en: mayo de 2007.

http://recursostic.javeriana.edu.co/wiki/index.php/Biblioteca_de_babel

Rebiun. Anuario de las bibliotecas universitarias y científicas españolas 2005. Madrid: Rebiun, 2006. 114 p. Consultado en: mayo de 2007. http://www.rebiun.org/doc/estadisticas/ANUARIO\%20REBIUN\%202005. $p d f$

Special Libraries Association (SLA). Consultado en: mayo de 2007. http://www.sla.org/

Universidad de Granada. Anuario 2005 Biblioteca Universitaria de Granada. Granada: UGR, 2006. 75 pp. Consultado en: mayo de 2007.

http://www.ugr.es/ biblio/biblioteca_ugr/anuarios/anuario_2005/index. html

Universidad de Granada. Grupo de repositorios de producción científica y visibilidad de la producción propia (Biblioteca Babel). Consultado en: mayo de 2007

http://www.ugr.es/ afporcel/repositorio1.htm

Universidad del Rosario. Biblioteca de Babel. Consultado en: mayo de 2007.

http://babel.urosario.edu.co/index.php

Universidad Metropolitana de Venezuela. Biblioteca de Babel. Consultado en: mayo de 2007.

http://agora.unimet.edu.vel

Universidad Técnica Federico Santa María. Estadísticas de biblioteca. Consultado en: mayo de 2007.

http://www.bib.utfsm.cl/2007/contenido/st.php

Universitäts-und Landesbibliothek. Impact and outcome of libraries. Consultado en: mayo de 2007.

http://www.ulb.uni-muenster.de/bibliothek/projekte/outcome.html

Atilio Bustos González, Director Sistema de Bibliote$c a$,

Pontificia Universidad Católica de Valparaíso, Av. Brasil 2950 - 2374631 Valparaíso, Chile.

Tel.: +56-32 22732 60; GSM: +56-99 32321 89; fax:

$+56-322273183$

abustos@ucv.cl

http://biblioteca.ucv.cl

http://agora.ucv.cl

http://ejbiotechnology.info

http://arpa.ucv.cl

http://margotloyola.ucv.cl

http://biblioteca.ucv.cl/atiliobustos/ 